\title{
Pneumomediastinum in a Heavy Weightlifter
}

\author{
Neeraja Swaminathan, Akanksha Agrawal, Pradhum Ram \\ Albert Einstein Medical Center, Philadelphia, PA, USA
}

Received: $12 / 03 / 2019$

Accepted: 03/04/2019

Published: 03/05/2019

How to cite this article: Swaminathan N, Agrawal A, Ram P. Pneumomediastinum in a heavy weightlifter. EJCRIM 2019;6: doi:10.12890/2019_001085.

Conflicts of Interests: The Authors declare that there are no competing interests.

This article is licensed under a Commons Attribution Non-Commercial 4.0 License

\section{ABSTRACT}

The presence of air in the mediastinal cavity (i.e., pneumomediastinum) is an uncommon condition. It is usually precipitated by an event such as penetrating trauma, barotrauma or increased intrathoracic pressure caused by severe vomiting or coughing. We present the case of a 32-year-old man who developed pneumomediastinum as a result of heavy weightlifting.

\section{LEARNING POINTS}

- Pneumomediastinum should be considered in the differential diagnosis of acute onset shortness of breath and chest pain.

- Pneumomediastinum can be precipitated without any obvious trauma as in cases of increased coughing/vomiting or heavy weightlifting.

- It is generally managed conservatively and resolves spontaneously.

\section{KEYWORDS}

Pneumomediastinum, weightlifting.

\section{CASE REPORT}

A 32-year-old male smoker presented to the emergency department with a 1-day history of dyspnoea and chest tightness. He denied chest pain, vomiting or drug use. He gave a history of lifting heavy weights during his workout sessions. On examination, he had an SpO2 of $86 \%$ with increased work of breathing, retractions, and was speaking in short interrupted sentences. He had diminished breath sounds on the left side. A chest radiograph to rule out pneumothorax was unremarkable. Administration of nebulization and steroids resulted in only marginal improvement as the patient continued to have tachypnoea and wheezing. In view of the persistent symptoms despite appropriate management, the patient underwent computed tomography (CT) of the chest. The CT revealed a pneumomediastinum causing bilateral supraclavicular subcutaneous emphysema without lesions in the lungs, trachea or oesophagus (Fig. 1). The patient was managed conservatively with serial CTs to check for progression, had an uneventful course and was discharged on day 3.

\section{DISCUSSION}

In a patient with acute onset dyspnoea and chest tightness, the common differential diagnoses are reactive airway disease, acute coronary syndrome and pulmonary embolism. A rarer differential diagnosis is pneumomediastinum, which should be considered especially if there is a history of heavy weightlifting. Pneumomediastinum is usually benign but can fatal if it progresses to mediastinitis or tension pneumothorax. The mediastinal cavity is the area enclosed by the pleura bilaterally, the thoracic inlet above and the diaphragm below. Following sudden changes in pressure, free air can enter this cavity as a result of the 'Macklin effect' which comprises alveolar rupture, tracking of air along the broncho-vasculature and spread of the pulmonary interstitial emphysema into the mediastinal cavity. Most patients with pneumomediastinum have chest pain, but the pathognomonic Hamman's crunch is only heard in about $18 \%$ of cases. 


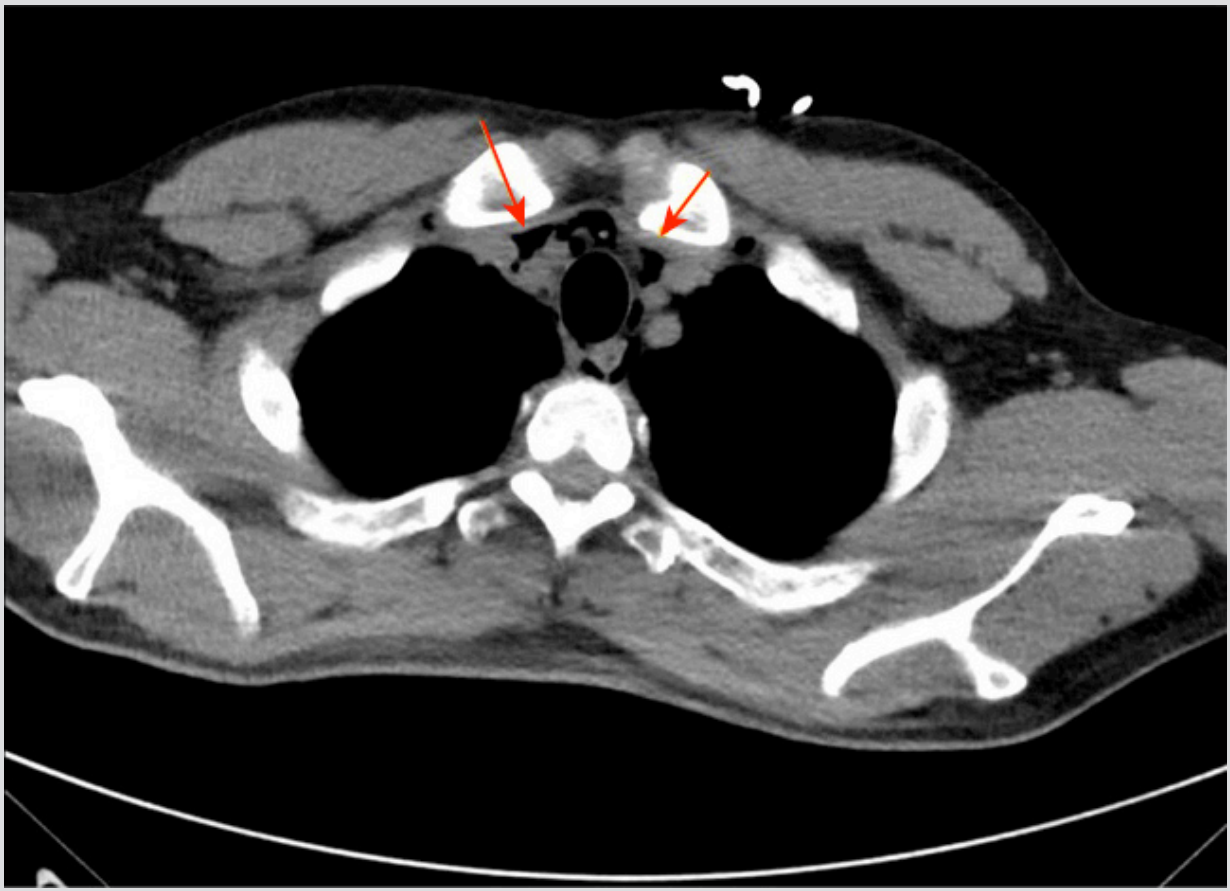

Figure 1. Non-contrast CT scan of the chest showing air in the mediastinal cavity (arrows) epiduritis

Other common symptoms include dyspnoea, cough, neck pain and dysphagia. The thymic sail sign due to elevation of the thymus by air in the mediastinum or the ring sign caused by air surrounding the pulmonary artery or its branches may also be visualized on imaging ${ }^{[1,2]}$.

Spontaneous pneumomediastinum is a rare entity, seen in approximately 1 in 30,000 emergency department referrals, with heavy weightlifting being a rarely reported cause or trigger. While pneumomediastinum can be dangerous, it is most often benign and tends to be over-treated. A swallow study is only indicated if there is suspicion of rupture of the aerodigestive tract. Intensive care, nil by mouth and intravenous antibiotics are only indicated if there is a diagnosis of oesophageal injury. A delay in the initiation of food consumption and waiting for the resolution of pneumomediastinum on imaging only prolongs hospital stay with no improvement in patient outcome ${ }^{[3]}$.

Pneumomediastinum is treated conservatively with rest, analgesics, oxygen and bronchodilators and does not recur in most cases. Administration of $100 \% \mathrm{O}_{2}$ can aid in resorption of free air and bed rest is recommended to expedite resolution of the condition. Patients are usually followed up on an outpatient basis with serial radiographic evaluations to confirm resolution of the pneumomediastinum ${ }^{[2]}$.

\section{REFERENCES}

1. Al-Mufarrej F, Badar J, Gharagozloo F, et al. Spontaneous pneumomediastinum: diagnostic and therapeutic interventions. J Cardiothorac Surg 2008;3:59.

2. Sahni S, Verma S, Grullon J, et al. Spontaneous pneumomediastinum: time for consensus. N Am J Med Sci 2013;5:460-464.

3. Macia I, Moya J, Ramos R, et al. Spontaneous pneumomediastinum: 41 cases. Eur J Cardiothorac Surg 2007;31:1110-1114. 